\title{
PROBLEMS OF NEUROHISTOLOGY
}

\section{Problems of Nervous Anatomy}

By Prof. J. Boeke. Pp. vii +164 . (London : Oxford University Press, 1940.) 7s. 6d. net.

$\mathrm{T}$ HE disadvantage of divorce between form and function in biological study is nowhere more often seen than in relation to the nervous system. Doubly valuable, therefore, is a review of some current problems by one of the foremost living neurohistologists who, while declaring that "however valuable physiological observations may be, as long as their real histological basis is disproportionately small or entirely missing, even the best physiological theories remain nebulous", is willing to admit that "morphological details everywhere only derive their full value in view of the physiological and functional insight which they are able to furnish".

The book is in four parts, based on as many lectures given in the latter part of 1937 in the Universities of London and Oxford and to the Anatomische Gesellschaft, with a bibliography which unfortunately does not include all names cited in the text. The first part deals with cutaneous innervation, and stresses the lack of a known structural basis for many clinical and experimental observations on cutaneous sensibility. Boeke rejects Head's view that two different kinds of nerve fibres are concerned with protopathic and epicritic sensation. In the light of recent histological, developmental and experimental studies, he suggests that protopathic sensation is the outcome of incompletely regenerated end-organs and as yet unmyelinated nerve fibres. He directs attention to the rich sympathetic plexus in the dermis, but the absence of all 'endings' in this plexus leads him to conclude that it is efferent in function.

In the next two parts the author develops a consideration of this "sympathetic ground plexus" in many parts of the body, and identifies the cellular elements in the plexus with the interstitial cells of Cajal. Boeke's review of the vexed question of the interstitial cells is one of the most useful things in his book. He thinks them to be of great phylogenetic significance in attempting to relate the vertebrate nervous system, with its highly organized cell patterns, to the simple nerve net of the lower invertebrates. This section was written before Prof. Woollard's last paper, in disproof of the invertebrate nerve net (Woollard and Harpman, J. Anat., 73 ; 1939). But Woollard's work, so sadly cut short, cannot be taken as conclusive, though it does imply that re-examination of the invertebrate nervous system is needed before further theoretical correlations are established. What neither the protagonists nor the antagonists of the nerve net seem to have considered is the relation between the "ground plexus" and the vascular system, which is undoubtedly retiform in its development and remains so in its periphery. Some of Boeke's own figures of the ground plexus strongly suggest a capillary relationship. How far is the one the trellis along which the other grows?

Several threads in the earlier sections are taken up in the last part, which is a review of the present state of the neurone doctrine in the light of modern physiological conceptions of the inter-neuronal synapse. It is a wise and thought-provoking chapter, which demands that out-worn concepts and conflicts be discarded so that fresh attacks can be made upon basic problems of neurology in the unhindered light of newer knowledge. Here, as elsewhere in the book, is evidence of the mature judgment, open mind, and breadth of vision of the man who has succeeded Cajal as the doyen of neurohistologists.

\section{NUTRITION IN WAR-TIME}

Nutrition and the War

By Dr. Geoffrey Bourne. Pp. xii + 126. (Cambridge: At the University Press, 1940.) $3 s .6 d$. net.

IN Great Britain there has been, in the last few years, a great improvement in the national dietary which has been accompanied by a corresponding improvement in national health. The limit of improvement has not yet been reached. The increase in physical fitness of recruits drawn from the poorer class after a few months in the Army and of slum children evacuated to the country is an indication of the extent to which national health and physical fitness can be still further increased. All we have learned of nutrition in the last twenty-five years suggests that food is probably the most important factor in this improvement in physical fitness. The author of this little book gives some striking illustrations of the connexion between diet and physical fitness. 